\title{
THE SQUAREROOT OF AN AMBIGUOUS FORM IN THE PRINCIPAL GENUS
}

\author{
by KENNETH HARDY* and KENNETH S. WILLIAMS $\dagger$
}

(Received 8th May 1991)

\begin{abstract}
A squareroot of an ambiguous form in the principal genus of primitive integral binary quadratic forms of fixed discriminant is given explicitly in terms of a solution of a certain Legendre equation.
\end{abstract}

1991 Mathematics subject classification: $11 \mathrm{E} 16$.

Let $D \equiv 0,1(\bmod 4)$ be a nonsquare integer. Let $f$ be a primitive, integral binary quadratic form of discriminant $D$, which is positive-definite if $D<0$. If $f$ belongs to the principal genus of classes of forms of discriminant $D$ then Gauss' famous duplication theorem (see for example [1, Theorem 4.21]) asserts that there exists a primitive binary quadratic form $g$ of discriminant $D$ such that $f \sim g^{2}$. Moreover Gauss [2, §286] has given a method of computing $g$ using the reduction of ternary quadratic forms. In [3] Shanks improves Gauss' method and provides an algorithm suitable for machine computation. In this note we show that when $f$ is an ambiguous form in the principal genus, $g$ can be described in a simple way in terms of the solution of a certain Legendre equation (eqn. (3) below).

Replacing $f$ by an equivalent form we may suppose that $f$ is of one of the following two types:

$$
f=A x^{2}+C y^{2}=(A, 0, C), \quad \mathrm{GCD}(A, C)=1, \quad D=-4 A C,
$$

or

$$
f=A x^{2}+A x y+C y^{2}=(A, A, C), \quad \operatorname{GCD}(A, C)=1, \quad D=A^{2}-4 A C
$$

We set

$$
\begin{cases}\alpha=2, B=C & , \text { if } f \text { is of type (I), } \\ \alpha=1, B=4 C-A, & \text { if } f \text { is of type (II) }\end{cases}
$$

so that

$$
D=-\alpha^{2} A B
$$

\footnotetext{
* Research supported by Natural Sciences and Engineering Research Council of Canada Grant A-8049. †Research supported by Natural Sciences and Engineering Research Council of Canada Grant A-7233.
} 
and show that there exist integers $X, Y, Z$ satisfying Legendre's equation

$$
A X^{2}+B Y^{2}=Z^{2}, \quad \operatorname{GCD}(X, Y)=1,
$$

with

$$
\begin{cases}\operatorname{GCD}(Z, 2 A B)=1 & , \text { if } f \text { is of type (I), } \\ \operatorname{GCD}(Z, 2 A B)=1 & \\ \quad \text { or } & \\ X \equiv Y \equiv Z+1 \equiv 1(\bmod 2), \operatorname{GCD}\left(\frac{Z}{2}, 2 A B\right)=1 & , \text { if } f \text { is of type (II). }\end{cases}
$$

To see this, recall that a form in the principal genus represents primitively a square coprime with any given integer. Thus, if $f$ is of type (I), there exist integers $X, Y, Z$ such that

$$
A X^{2}+C Y^{2}=Z^{2}, \quad \operatorname{GCD}(X, Y)=1, \quad \operatorname{GCD}(Z, 2 A C)=1,
$$

establishing (3) and (4) in this case. If $f$ is of type (II) there exist integers $R, S, T$ such that

$$
A R^{2}+A R S+C S^{2}=T^{2}, \quad \operatorname{GCD}(R, S)=1, \quad \operatorname{GCD}(T, 2 A(4 C-A))=1
$$

Set

$$
\begin{aligned}
& X=R+\frac{S}{2}, \quad Y=\frac{S}{2}, \quad Z=T, \quad \text { if } S \text { is even, } \\
& X=2 R+S, \quad Y=S, \quad Z=2 T, \quad \text { if } S \text { is odd }
\end{aligned}
$$

The integers $X$ and $Y$ satisfy

$$
A X^{2}+(4 C-A) Y^{2}=Z^{2}, \quad \operatorname{GCD}(X, Y)=1,
$$

with

$$
\operatorname{GCD}(Z, 2 A(4 C-A))=1, \quad \text { if } S \text { is even, }
$$

or

$$
X \equiv Y \equiv Z+1 \equiv 1(\bmod 2), \operatorname{GCD}(Z / 2,2 A(4 C-A))=1, \quad \text { if } S \text { is odd, }
$$

establishing (3) and (4) in this case. From (3) and (4) we easily deduce that

$$
\operatorname{GCD}(A, Y)=\operatorname{GCD}(B, X)=\operatorname{GCD}(X, Z)=\operatorname{GCD}(Y, Z)=1 \text {. }
$$

Let $u, v$ be integers such that

$$
X v-Y u=1 .
$$

When $f$ is of type (II) and $Z \equiv 1(\bmod 2)$, we can arrange that $u$ and $v$ are both odd by replacing $(u, v)$ by $(u+X, v+Y)$, if necessary, as $X$ and $Y$ are of opposite parity.

We define $a, b, c$ by 


$$
\left.\begin{array}{ll}
\begin{array}{l}
a=Z, b=2(A X u+B Y v), c=A u^{2}+B v^{2} \\
a=Z, b=A X u+B Y v, c=\left(A u^{2}+B v^{2}\right) / 4
\end{array} & \begin{array}{c}
\text { if } f \text { is of type (I), } f \text { is of type (II), } \\
\text { and } Z \equiv 1(\bmod 2),
\end{array} \\
a=Z / 2, b=A X u+B Y v, c=A u^{2}+B v^{2} & \begin{array}{c}
\text { if } f \text { is of type (II) } \\
\text { and } Z \equiv 0(\bmod 2)
\end{array}
\end{array}\right\}
$$

Note that when $f$ is of type (II) and $Z \equiv 1(\bmod 2)$ we have

$$
c=A \frac{\left(u^{2}-v^{2}\right)}{4}+C v^{2},
$$

which is an integer as both $u$ and $v$ are odd in this case. Thus the quantities $a, b, c$ in (7) are all integers.

We define the integral binary quadratic form $g$ by

$$
g=(a, b, a c)
$$

and prove:

Theorem. $g^{2} \sim f$.

Proof. We first show that $g=(a, b, a c)$ is a primitive form, that is

$$
\operatorname{GCD}(a, b)=1 \text {. }
$$

We have

$$
\begin{array}{rlrl}
b Y & =\alpha(A X u+B Y v) Y & & (\text { by }(1),(7)) \\
& =\alpha\left(A X Y u+\left(Z^{2}-A X^{2}\right) v\right) & & (\text { by (3)) } \\
& =\alpha\left(Z^{2} v-A X(X v-Y u)\right) & \\
& =\alpha\left(Z^{2} v-A X\right) & & (\text { by }(6))
\end{array}
$$

so that

$$
\begin{array}{rlrl}
\operatorname{GCD}(a, b) & =\operatorname{GCD}(a, b Y) & & (\text { by }(5),(7)) \\
& =\operatorname{GCD}\left(a, \alpha\left(Z^{2} v-A X\right)\right) \\
& =\operatorname{GCD}\left(a, Z^{2} v-A X\right) & & (\text { by }(1),(4),(7)) \\
& =\operatorname{GCD}(a, A X) & & (\text { by }(7)) \\
& =1 & & (\text { by }(4),(5),(7))
\end{array}
$$

as claimed. 
Next we show that $g=(a, b, a c)$ has discriminant $D$. We have, appealing to (2), (3), (6) and (7),

$$
\begin{aligned}
b^{2}-4 a^{2} c & =\alpha^{2}\left((A X u+B Y v)^{2}-Z^{2}\left(A u^{2}+B v^{2}\right)\right) \\
& =\alpha^{2}\left((A X u+B Y v)^{2}-\left(A X^{2}+B Y^{2}\right)\left(A u^{2}+B v^{2}\right)\right) \\
& =-\alpha^{2} A B(X v-Y u)^{2} \\
& =-a^{2} A B \\
& =D
\end{aligned}
$$

Finally we observe that the unimodular transformation with matrix

$$
\begin{aligned}
& {\left[\begin{array}{ll}
X & u \\
Y & v
\end{array}\right], \text { if } f \text { is of type (I) }} \\
& {\left[\begin{array}{cc}
X-Y & \frac{u-v}{2} \\
2 Y & v
\end{array}\right], \text { if } f \text { is of type (II) and } Z \equiv 1(\bmod 2),} \\
& {\left[\begin{array}{cc}
\frac{X-Y}{2} & u-v \\
Y & 2 v
\end{array}\right], \text { if } f \text { is of type (II) and } Z \equiv 0(\bmod 2),}
\end{aligned}
$$

transforms $f$ into the form $\left(a^{2}, b, c\right)$. Hence, in view of (9) (see [1, Corollary 4.13]), we have

$$
f \sim\left(a^{2}, b, c\right) \sim(a, b, a c)^{2}=g^{2}
$$

as asserted.

Example 1. The ambiguous form $f=(401,0,419)$ has discriminant $D=-67276=$ $-4 \cdot 401 \cdot 419 \equiv 20(\bmod 32)$ so its generic characters are the Legendre symbols $(\overline{401})$ and $(\overline{419})$. The form $f$ represents primitively the odd integer $401 \cdot 2^{2}+419=2023$, which is coprime with $D$. As

$$
\left(\frac{2023}{401}\right)=\left(\frac{18}{401}\right)=\left(\frac{2}{401}\right)=1
$$

and

$$
\left(\frac{2023}{419}\right)=\left(\frac{-72}{419}\right)=\left(\frac{-2}{419}\right)=1
$$


the form $f$ lies in the principal genus of classes of primitive, positive-definite binary quadratic forms of discriminant $D$. The appropriate Legendre equation is

$$
401 X^{2}+419 Y^{2}=Z^{2}
$$

which must have an integral solution $(X, Y, Z) \neq(0,0,0)$ satisfying (see [4])

$$
0 \leqq X \leqq \sqrt{419}=20, \quad 0 \leqq Y \leqq \sqrt{401}=20 .
$$

A simple computer search quickly finds

$$
X=11, \quad Y=4, \quad Z=235 .
$$

A solution of

$$
11 v-4 u=1
$$

is

$$
u=-3, \quad v=-1,
$$

so, by (7) and (8), a squareroot of $f=(401,0,419)$ is given by

$$
g=(235,-29818,946580) \sim(235,-208,761) .
$$

Example 2. The ambiguous form $f=(5849,5849,2925)$ has discriminant $D=$ $-34222499=-5849 \cdot 5851 \equiv 1(\bmod 4)$ so its generic characters are $(\overline{5849})$ and $(\overline{5851})$. The form $f=(5849,5849,2925)$ represents primitively the odd integer 2925 which is coprime with the discriminant $D$. As $\left(\frac{2925}{5849}\right)=\left(\frac{5849}{2925}\right)=\left(\frac{-1}{2925}\right)=1$ and $\left(\frac{2925}{5851}\right)=\left(\frac{5851}{2925}\right)=$ $\left(\frac{1}{2925}\right)=1$ the form $f$ belongs to the principal genus. The appropriate Legendre equation is

$$
5849 X^{2}+5851 Y^{2}=Z^{2}
$$

which has the solution

$$
X=3, \quad Y=5, \quad Z=446 .
$$

A solution of

$$
3 v-5 u=1
$$

is

$$
u=1, \quad v=2
$$

so, by (7) and (8), a squareroot of $f$ is given by

$$
g=(223,76057,6523419) \sim(223,-209,38415) .
$$




\section{REFERENCES}

1. Duncan A. Buell, Binary Quadratic Forms (Springer-Verlag, New York, 1989).

2. Carl Friedrich Gauss, Disquisitiones Arithmeticae (Yale University Press, 1966).

3. Daniel Shanks, Gauss's ternary form reduction and the 2-Sylow subgroup, Math. Comp. 25 (1971), 837-853. (Corrigendum, Math. Comp. 32 (1978), 1328-1329.)

4. Kenneth S. Williams, On the size of a solution of Legendre's equation, Utilitas Math. 34 (1988), 65-72.

Department of Mathematics and Statistics

Carleton University

Ottawa, Ontario

Canada K1S 5B6 